\title{
Ising tricriticality and the dilute $\mathrm{A}_{3}$ model.
}

\author{
Katherine A Seaton \\ School of Mathematical and Statistical Sciences, La Trobe University, Victoria 3086, \\ Australia
}

\begin{abstract}
Some universal amplitude ratios appropriate to the $\phi_{2,1}$ perturbation of the $c=\frac{7}{10}$ minimal field theory, the subleading magnetic perturbation of the tricritical Ising model, are explicitly demonstrated in the dilute $A_{3}$ model, in regime 1.
\end{abstract}

AMS classification scheme numbers: 82B23, 81T40

\section{Introduction}

While the idea that universality should be described not only by referring to critical exponents but also to universal amplitude ratios is not new, it continues to attract interest. Universal ratios are constructed in such a way as to cause cancellation of any metric factors associated with the particular realization of a universality class being considered. Thus comparison of these ratios, say from a field theory and a lattice model, or as measured in an experimental system, increases our understanding of universality. Alternatively, they may be considered to have predictive value.

Since the time of the comprehensive review of universal critical-point amplitude ratios in [四], there have been important developments in integrable quantum field theory. Those most pertinent to this paper are described below in Section 3. Amplitude ratios for the universality classes of the $q$-state Potts model [2] and the tricritical Ising model [3, [4] have recently been constructed using field theoretic approaches. For the Ising model in a magnetic field, transfer matrix results for a lattice Hamiltonian are compared with $S$-matrix determination of universal amplitudes in [5].

Probably better recognized for its connection to the Ising model in a magnetic field, in this short paper the dilute A model [6] is discussed in relation to the subleading magnetic perturbation of tricritical Ising model. That model is introduced in Section 2 and some quantum field theory results applying to it are outlined in Section 3 . In Section 1 the dilute $\mathrm{A}_{3}$ model, and some results for it, are described; it is shown to be a solvable lattice model realization of the $\phi_{2,1}$ perturbation of $\mathcal{M}(4,5)$. Motivated by [4], universal amplitude ratios are constructed in Section 5. These are found to be in perfect agreement with results from quantum field theory, which have appeared since the original solution of the dilute A model in [7]. A brief discussion concludes the paper. 


\section{The tricritical Ising model}

The tricritical Ising model corresponds to the minimal unitary conformal field theory

$\mathcal{M}(4,5)$ with central charge $c=\frac{7}{10}$ and its critical exponents are related to the conformal weights

$$
\Delta_{r, s}^{(5)}=\frac{(5 r-4 s)^{2}-1}{80}, \quad 1 \leqslant r \leqslant 3,1 \leqslant s \leqslant 4 .
$$

One lattice realization of this model is the Blume-Capel model [8], a spin-1 generalization of the Ising model with vacancies favoured by a chemical potential $\mu$. The Hamiltonian can be written as

$$
\mathcal{H}_{\mathrm{BC}}=-J \sum_{\langle i, j\rangle} s_{i} s_{j}-H \sum_{i=1}^{N} s_{i}+\mu \sum_{i=1}^{N}\left(s_{i}\right)^{2},
$$

where $J$ is the nearest-neighbour coupling and $H$ is an external magnetic field. The lattice variable may be thought of as

$$
s_{i}=\sigma_{i} v_{i} \quad \text { where } \quad \sigma_{i}= \pm 1, v_{i}=0,1 .
$$

The spin is $\sigma_{i}$ and the occupancy (or vacancy) is expressed by $v_{i}$. The model further admits a subleading or staggered magnetic field, in which case it is more properly called the Blume-Emery-Griffiths model:

$$
\mathcal{H}_{\mathrm{BEG}}=\mathcal{H}_{\mathrm{BC}}-H_{3} \sum_{\langle i, j\rangle} v_{i} v_{j}\left(\sigma_{i}+\sigma_{j}\right) .
$$

The staggered field favours aligned spins on neighbouring sites. The phase diagram of this model is like that of $\mathrm{He}^{3}-\mathrm{He}^{4}$ mixtures shown in 90. The features of interest for this paper are its wings of two-phase coexistence, on one of which the phases are $s_{i}=0$ and $s_{i}=1$, and on the other $s_{i}=0$ and $s_{i}=-1$. The subleading magnetic perturbation of the tricritical Ising model gives access to a one-dimensional section of the full three-dimensional two-phase coexistence manifold [10].

\section{The subleading magnetic perturbation}

Two remarkable advances in quantum field theory should be mentioned in the context of the tricritical Ising model. Zamolodchikov showed that the minimal conformal field theories $\mathcal{M}\left(p, p^{\prime}\right)$ admit integrable perturbations by one of the operators $\phi_{1,2}, \phi_{1,3}, \phi_{1,5}$ and $\phi_{2,1}$, if they are relevant [11, 12]. It was further established, using the connection of the quantum field theories to scattering theory, that the minimal unitary theories $\mathcal{M}(p, p+1)$ for $p=3,4,5$ perturbed by $\phi_{1,2}$ correspond to the exceptional $\mathrm{E}_{6}, \mathrm{E}_{7}$ and $\mathrm{E}_{8}$ Toda field theories [12, [13].

An important study of the mass spectra and phase diagrams of the four relevant perturbations of the tricritical Ising model was undertaken in [10] using the truncated conformal space approach. The two thermal perturbations are $\phi_{1,2}$ (leading) and $\phi_{1,3}$ (which is related to the chemical potential $\mu$ in (2)). Interestingly, the 'usual' magnetic 
Table 1. The four perturbations of the tricritical Ising model

\begin{tabular}{llll}
\hline Field & Perturbation & Weight & Lattice model \\
\hline$\varphi_{1}$ Leading magnetic & $\phi_{2,2}$ & $\frac{3}{80}$ & Not integrable \\
$\varphi_{2}$ Leading thermal & $\phi_{1,2}$ & $\frac{1}{10}$ & Dilute $\mathrm{A}_{4}$, regime 2 \\
$\varphi_{3}$ Subleading magnetic & $\phi_{2,1}$ & $\frac{7}{16}$ & Dilute $\mathrm{A}_{3}$, regime 1 \\
$\varphi_{4}$ Subleading thermal & $\phi_{1,3}$ & $\frac{3}{5}$ & ABF $\mathrm{A}_{4}$, regime III \\
\hline
\end{tabular}

field $H$ in (2) corresponds to the perturbation $\phi_{2,2}$ of $\mathcal{M}(4,5)$ which is relevant but not integrable, while the staggered magnetic field in (4) corresponds to the integrable perturbation $\phi_{2,1}$, though it is not realizable in a laboratory [9]. It is conceptually and notationally convenient [10] to number the fields according to Landau-Ginzburg $\Phi^{6}$ formulation, from most relevant $\left(\varphi_{1}\right)$ to least relevant $\left(\varphi_{4}\right)$ on the basis of the associated conformal weights (1). Table 11 summarises this and what is to follow about the manifestations of these four perturbations.

The $S$-matrix for the subleading magnetic perturbation in particular was studied in [14, 15], and using the thermodynamic Bethe ansatz in [16]. Mass spectra in general are exactly related to bulk energies and coupling constants of the appropriate perturbations in [17]. Degeneracy in the spectrum for $\phi_{2,1}$ means that only one distinct mass value emerges.

Other results for the $\phi_{2,1}$ perturbation of $\mathcal{M}(p, p+1)$ can also be found among general discussions of integrable perturbed quantum field theories. In particular, expressions for one-point correlation functions or vacuum expectation values of the local fields are proposed in [18]. Explicit values are given for the tricritical Ising model, and compared to numerical results of [19]. Predictably, two degenerate but asymmetric groundstates are identified for each sign of the coupling constant.

Universal amplitude ratios for the relevant perturbations of the tricritical Ising model have recently been constructed [3, 4], by collecting together information from the approaches above, as well as numerical results. These ratios involve the amplitudes of the correlation lengths $\xi_{i}$ and of the free energy $f_{i}$ (where the subscript $i=1, \ldots, 4$ denotes the field taking the model off-critical) but are independent of the coupling constants $g_{i}$ and hence of any nonuniversal metric factors. Further, they involve the amplitudes of the vacuum expectations of the local fields

$$
\left\langle\varphi_{j}\right\rangle_{i} \equiv B_{j i} g_{i}^{\Delta_{j} /\left(1-\Delta_{i}\right)},
$$

as well as generalized susceptibilities. Some of these ratios should be observable both in real-world representations of this universality class, and the related solvable lattice models, provided the physical quantities required can be measured or constructed.

Each of the three integrable perturbations of the tricritical Ising model has been identified with a particular solvable lattice model in its massive, scaling limit. The elliptic nome of the $\mathrm{A}_{4} \mathrm{ABF}$ model [20, 21] in regime III corresponds to the coupling constant of the subleading thermal perturbation, $\phi_{1,3}$. Smirnov [22] conjectured that 
lattice models for $\phi_{1,2}$ and $\phi_{2,1}$ would be based on RSOS restriction of the IzerginKorepin [23] $A_{2}^{(2)} R$-matrix. The dilute A model [6] is a hierarchy of such models, and provides the two remaining cases. Of these, most attention has focused on the dilute $\mathrm{A}_{4}$ model in regime 2 , which is in the universality class of the $\phi_{1,2}$ perturbation, to elucidate the hidden $\mathrm{E}_{7}$ structure [24, 25, 26, 27]. A detailed description of how the dilute $\mathrm{A}_{3}$ model in regime 1 realizes the $\phi_{2,1}$ perturbation of the tricritical Ising model will follow in Section 4. Delfino 28] gives an interesting phase diagram concerning the points of contact of the regimes of these three solvable models from a discussion of double perturbations of the minimal unitary series by $\phi_{1,3}$ and one of $\phi_{1,2}$ or $\phi_{2,1}$.

\section{The dilute $A_{3}$ model in regime 1 as the tricritical Ising model}

The dilute $\mathrm{A}_{L}$ model [6] is a hierarchy of solvable restricted solid-on-solid models, defined on the square lattice and labelled by the allowed number of heights, $L$. The adjacency condition of the model is that neighbouring sites of the lattice are either occupied by the same height or differ by one. The face weights of the model are parameterized using elliptic functions. It is solvable in four off-critical regimes, generated by the elliptic nome $p$ and the value of the crossing parameter, which in regime 1 团 is

$$
\lambda=\frac{\pi}{4}\left(\frac{L}{L+1}\right),
$$

while the central charge, known from equivalence with the $\mathrm{O}(n)$ model, for regime 1 is

$$
c=1-\frac{6}{(L+2)(L+1)}
$$

For odd $L$ the elliptic nome breaks the $\mathbb{Z}_{2}$ symmetry of the underlying adjacency diagram and is thus magnetic-field like; changing the sign of $p$ causes height relabelling

$a \rightarrow L+1-a$, where $a=1, \ldots, L$. It is sometimes necessary to distinguish between regime $1^{+}$where $p>0$ and regime $1^{-}$where $p<0$.

The singular part of the free energy of the dilute $A_{L}$ model in regime 1 to leading order as the nome $p \rightarrow 0$ behaves as

$$
f_{\mathrm{s}} \sim \mathcal{A}_{L} p^{\frac{4}{3}\left(\frac{L+1}{L}\right)}
$$

which follows from the partition function-per-site calculated either using the inversion relation method [7] or the largest eigenvalue of the row-to-row transfer matrix [29]. For odd $L$ this gave [7] the critical exponent $\delta=3 L /(L+4)$ and the associated scaling dimension

$$
\Delta_{p}=\frac{1}{1+\delta}=\frac{L+4}{4(L+1)}=\Delta_{2,1}^{(L+2)}
$$

so that in regime 1 the nome generates perturbation of the unitary minimal model $\mathcal{M}(L+1, L+2)$ by the operator $\phi_{2,1}$. This is the basis of the earlier assertion that for $L=3$ the nome relates to the subleading magnetic perturbation of the tricritical Ising

$\ddagger$ This regime labelling differs from [6] but is followed in [7] and all subsequent papers. 
model; or in field theoretic notation, the coupling constant $g_{3}$ of $\varphi_{3}$; or in the language of the lattice Hamiltonian, the staggered magnetic field.

Correspondence can also be drawn at the level of details of the groundstates. In regime 1 the $\mathrm{A}_{3}$ model has two ferromagnetic groundstates in the ordered $(|p| \rightarrow 1)$ limit, for each sign of the elliptic nome [0]. For one sign of $p$ all sites of the lattice are occupied either by height 1 , or by height 2 . Under the field reversal noted before, the groundstates for the other sign of $p$ consist of all lattice sites being occupied by height 3 or by height 2 . The straightforward identification of the dilute $\mathrm{A}_{3}$ model height variable $a_{i}$ at site $i$ with the spin and vacancy variables (3) via

$$
a_{i}=2+\sigma_{i} v_{i}
$$

maps the groundstates to those described in Section 2. The adjacency condition of the dilute $\mathrm{A}_{3}$ model then corresponds to each occupied site having as its neighbour either an empty site, or an aligned spin.

Local height probabilities $P^{b c}(a)$, expressing the probability that a site deep in the lattice is occupied by height $a$ when the model is in the phase labelled by $(b, c)$ were calculated for the dilute $\mathrm{A}_{L}$ model $(L$ odd) in [7], using the corner transfer matrix technique [30. This calculation involves considering a series of finite square lattices with boundary sites set to the configurations $(b, c)$, solving recurrence relations and then taking the lattice size to infinity. The polynomials generated in this process for general $L$ in regime 1 have found application as the required finitizations of the bosonic side of Rogers-Ramanujan-type identities, termed $\phi_{2,1}$ polynomial identities [31.

Following Huse [21], generalized order parameters

$$
R_{k}^{b c}=\sum_{a=1}^{L} \frac{\sin ((k+1) a \pi /(L+1))}{\sin (a \pi /(L+1))} P^{b c}(a) \quad k=0, \ldots L-1
$$

were defined and the leading order behaviour determined [7]. For $L=3$ in regime $1^{+}$, where the ferromagnetic groundstates have $c=b$,

$$
R_{k}^{b b} \sim p^{\left[(k+1)^{2}-1\right] / 45} \sin ((k+1) \pi s / 5) / \sin (\pi s / 5) ; \quad s= \begin{cases}1 & \mathrm{~b}=1 \\ 3 & \mathrm{~b}=2 .\end{cases}
$$

The associated scaling dimensions were determined using (14), (5) and (6) to be

$$
\Delta_{k}=\Delta_{k+1, k+1}^{(5)} \quad k=1,2 .
$$

Using the property $\Delta_{r, s}^{(5)}=\Delta_{4-r, 5-s}^{(5)}, R_{1}^{b b}$ is identified with the expectation value of the operator $\phi_{2,2}$ (or $\varphi_{1}$ ) and similarly, $R_{2}^{b b}$ with $\phi_{1,2}$ (or $\varphi_{2}$ ), in both of the two phases labelled by $b$. (Fairly trivially, $R_{0}^{b b}=1$ by definition, and corresponds to $\phi_{1,1}$ or the identity operator.)

The variable $s$ introduced in (7) depends only on the height $b$, and provides the identification of the lattice model groundstates and the phase labelling in [18] required if we are to proceed to compare universal quantities. In the field theory context the phases are $\left|0_{s}\right\rangle$ with $s=2,4$ for positive coupling constant, and $s=1,3$ for negative coupling constant. (For reasons to do with arbitrary choices made in the Boltzmann weights of 
the dilute A model as set up in [7] the elliptic nome is actually $-g_{3}$, so that regime $1^{+}$ corresponds to $g_{3}<0$. Applying the appropriate height reversal to the working leading to (7) one obtains $s=2,4$ in regime $1^{-}$. Since the perturbation is magnetic, the ratios to be considered in Section 5 are in fact unchanged by this rather technical detail. A similar observation is made for dilute $\mathrm{A}_{4}$ in [25, 27], where $p<0$ is noted to be the high temperature regime.)

\section{Universal amplitude ratios}

Knowing just $\left\langle\varphi_{j}\right\rangle_{3}, f_{3}$ and $\xi_{3}$ (see (11) below) does not permit construction of many of the various amplitude ratios proposed in [4. However, we can consider the ratio of $\left\langle\varphi_{j}\right\rangle_{3}$ in one phase of the tricritical Ising model (say $s=-1$ ) with the expectation value of the same operator in the coexisting phase $(s=0)$, for $j=1,2,3$ :

$$
\frac{\left\langle\varphi_{j}\right\rangle_{3}^{(-)}}{\left\langle\varphi_{j}\right\rangle_{3}^{(0)}}=\frac{B_{j 3}^{(-)}}{B_{j 3}^{(0)}}
$$

From (5), these ratios are pure numbers independent of the nome or $g_{3}$, and hence of any associated microscopic scaling factors of the solvable lattice realization of the tricritical Ising model, or of the field theory considered in [18].

First, $\left\langle\varphi_{3}\right\rangle_{3}=-\frac{\partial f_{3}}{\partial g_{3}}$, and since the amplitude of the free energy expression does not depend on the sign of the nome or on the phase, $B_{33}^{(-)} / B_{33}^{(0)}=1$, in agreement with the identical numerical values given in Table XVII of [1].

From (7) we find

$$
\frac{B_{j 3}^{(-)}}{B_{j 3}^{(0)}}=\frac{R_{j}^{11}}{R_{j}^{22}}=\frac{1+\sqrt{5}}{1-\sqrt{5}} \quad j=1,2 .
$$

In the expressions for $\left\langle 0_{s}\left|\phi_{l, k}\right| 0_{s}\right\rangle$ given in [18] the only $s$ or phase dependent factor is

$$
\sin (\pi s|5 l-4 k| / 5) / \sin (\pi s / 5)
$$

which should be compared to the coefficients in (7). The amplitude ratios constructed from the field theory are then in perfect accord with (9):

$$
\frac{\left\langle 0_{1}\left|\phi_{1,2}\right| 0_{1}\right\rangle}{\left\langle 0_{3}\left|\phi_{1,2}\right| 0_{3}\right\rangle}=\frac{\left\langle 0_{1}\left|\phi_{2,2}\right| 0_{1}\right\rangle}{\left\langle 0_{3}\left|\phi_{2,2}\right| 0_{3}\right\rangle}=\frac{1+\sqrt{5}}{1-\sqrt{5}} \text {. }
$$

One further universal amplitude can be calculated. The single correlation length of the model in regime 1 (corresponding to one distinct mass as mentioned in Section 3) was calculated from the leading transfer matrix eigenvalue excitation, constructed by Baxter's exact perturbative method [30] using the Bethe ansatz equations [32], in 29, 33. From the general expression for all $L$ in terms of standard elliptic theta functions (following the approach taken in [34]),

$$
\xi^{-1}=2 \log \left[\frac{\vartheta_{4}\left(\frac{\pi}{12}, p^{\pi / 6 \lambda}\right)}{\vartheta_{4}\left(\frac{5 \pi}{12}, p^{\pi / 6 \lambda}\right)}\right]=8 \sum_{n=1}^{\infty} \frac{1}{n} \frac{\left(p^{\pi / 6 \lambda}\right)^{n}}{1-\left(p^{\pi / 6 \lambda}\right)^{2 n}} \sin \left(\frac{n \pi}{2}\right) \sin \left(\frac{n \pi}{3}\right),
$$


we now obtain the coefficient of the leading order term for $L=3$ to be

$$
\xi^{-1} \sim 4 \sqrt{3} p^{8 / 9}+\mathrm{O}\left(\left(p^{8 / 9}\right)^{3}\right) .
$$

Combining this with the free energy amplitude $\mathcal{A}_{3}=2 \sqrt{3} / \cos (4 \pi / 9)$ gives the tricritical Ising subleading magnetic amplitude

$$
f_{\mathrm{s}} \xi^{2}=\frac{1}{8 \sqrt{3} \cos \left(\frac{4 \pi}{9}\right)},
$$

in agreement with the expression for it in [17]. This universal quantity is related to $R_{\xi}^{3}$ of [四].

\section{Discussion}

Only some of the objects used in constructing universal amplitude ratios in [四] can be calculated in the dilute $\mathrm{A}_{3}$ 'version' of the tricritical Ising model considered in this paper. To utilize what is available, a different universal quantity (8) has been considered. Thus equations (9) and (12) go some way towards answering a challenge put out in [3], to independently determine univeral amplitude ratios for the tricritical Ising model from a solvable lattice formulation. One barrier to constructing similar ratios to (8) for the dilute $\mathrm{A}_{4}$ model in regime 2 , which corresponds to the leading thermal perturbation, is that local height probabilities for $L$ even have not yet been calculated (in any regime). However, amplitudes akin to (12] are discussed for dilute $\mathrm{A}_{4}$ in regime 2 in [27], where the conjectured [25] $\mathrm{E}_{7}$ mass ratios are confirmed. There are more general observations

to be made along the lines of this paper, and it is intended to report on them in the near future.

\section{Acknowledgments}

This paper was written while the author enjoyed the hospitality of the Department of Mathematics and Statistics at the University of Melbourne. She also acknowledges the importance to it of earlier collaboration on the results re-examined here with Bernard Nienhuis, Paul Pearce and Ole Warnaar, and recent work with Murray Batchelor.

\section{References}

[1] Privman V, Hohenberg P C and Aharony A 1991 Phase Transitions and Critical Phenomena vol. 14 C Domb and J L Lebowitz, eds (London: Academic Press)

[2] Delfino G and Cardy J L 1998 Nucl. Phys. B 519 551-78

[3] Fioravanti D, Mussardo G and Simon P 2000 Phys. Rev. Lett. 85 126-9

[4] Fioravanti D, Mussardo G and Simon P 2000 Phys. Rev. E 63016103

[5] Caselle M and Hasenbusch M 2000 Nucl. Phys. B 579 667-703

[6] Warnaar S O, Nienhuis B and Seaton K A 1992 Phys. Rev. Lett. 69 710-712

Warnaar S O, Nienhuis B and Seaton K A 1993 Int. J. Mod. Phys. B 7 3727-36

[7] Warnaar S O, Pearce P A, Seaton K A and Nienhuis B 1994 J. Stat. Phys. 74 469-531

[8] Blume M 1966 Phys. Rev. 141517 -524 
Capel H 1966 Physica A 32 966-988

Blume M, Emery V J and Griffiths R B 1971 Phys. Rev. A 4 1071-1077

[9] Griffiths R B 1970 Phys. Rev. Lett. 24 715-717

[10] Lässig M, Mussardo G and Cardy J L 1991 Nucl. Phys. B 348 591-618

[11] Zamolodchikov A B 1987 JETP Lett. 46 160-4

[12] Zamolodchikov A B 1989 Adv. Stud. Pure Math. 19 641-74

[13] Zamolodchikov A B 1989 Int. J. Mod. Phys. A 4 4235-48

Fateev V A and Zamolodchikov A B 1990 Int. J. Mod. Phys. A 5 1025-48

[14] Colomo F, Koubek A and Mussardo G 1992 Phys. Lett. B 274 367-73

Colomo F, Koubek A and Mussardo G 1992 Int. J. Mod. Phys. A 7 5281-305

[15] Klassen T R and Melzer E 1992 Nucl. Phys. B 382 441-85

[16] Ellem R M and Bazhanov V V 1998 Nucl. Phys. B 512 563-80

[17] Fateev V A 1994 Phys. Lett. B 324 45-51

[18] Fateev V, Lukyanov S, Zamolodchikov A B and Zamolodchikov Al B 1998 Nucl. Phys. B 516 $652-74$

[19] Guida R and Magnoli N 1997 Phys. Lett. B 411 127-33

[20] Andrews G E, Baxter R J and Forrester P J 1984 J. Stat. Phys. 35 193-266

[21] Huse D A 1984 Phys. Rev. B 30 3908-15

[22] Smirnov F A 1991 Int. J. Mod. Phys. A 6 1407-28

[23] Izergin A G and Korepin V E 1981 Commun. Math. Phys. 79 303-16

[24] Warnaar S O and Pearce P A 1996 Int. J. Mod. Phys. A 11 291-311

[25] Batchelor M T and Seaton K A 1998 Eur. Phys. J. B 5 719-25

Seaton K A and Batchelor M T 1999 Group22: Proc. of the XXII Int. Colloq. on Group Theoretical Methods in Physics S P Corney, R Delbourgo and P D Jarvis, eds. (Cambridge MA: International Press) pp 274-8

[26] Suzuki J 2000 Physical Combinatorics M Kashiwara and T Miwa, eds. (Boston, Cambridge MA: Birkhauser) pp 217-47

[27] Seaton K A and Batchelor M T in preparation for submission to J. Math. Phys.

[28] Delfino G 2000 Nucl. Phys. B 585 597-613

[29] Batchelor M T and Seaton K A 1997 J. Phys. A: Math. Gen. 30 L479-84

[30] Baxter R J 1982 Exactly Solved Models in Statistical Mechanics (London: Academic Press)

[31] Berkovich A and McCoy B M 2000 Ramanujan Journal 4 353-83

Berkovich A, McCoy B M and Pearce P A 1998 Nucl. Phys. B 519 597-625

[32] Bazhanov V V, Nienhuis B and Warnaar S O 1994 Phys. Lett. B 322 198-206

[33] Batchelor M T and Seaton K A 1998 Nucl. Phys. B 520 697-744

[34] Seaton K A and Batchelor M T 2001 J. Stat. Phys. 102 1019-27 